\title{
La protection des personnes selon les directives éthiques internationales du CIOMS
}

\section{Michel B. Vallotton}

Professeur honoraire de la Faculté de Médecine, ancien chef de la Division d'Endocrinologie et Diabétiologie du Département de Médecine de la Faculté de Médecine de Genève, Président du Conseil des Organisations Internationales des Sciences Médicales (CIOMS)

Correspondance:

Prof. Dr Michel B. Vallotton Centre Médical Universitaire 1 , rue Michel-Servet CH-1206 Genève

Tél. 0223795482

Fax 0223795479

michel.vallotton@unige.ch
Le Conseil des Organisations Internationales des Sciences Médicales (CIOMS) est une organisation non-gouvernementale créée en 1949 conjointement par l'Organisation Mondiale de la Santé (OMS) et l'UNESCO. Il a donc célébré l'année passée son soixantième anniversaire. Ses tâches relèvent de deux domaines: d'une part la sécurité des produits pharmaceutiques, d'autre part l'éthique dans la recherche médicale.

Le premier domaine regroupe plusieurs thèmes abordés par des groupes de travail qui publient régulièrement leurs rapports. Ce sont: le traitement des informations de sécurité tirées des essais cliniques, le développement des mises à jour de la sécurité pharmacologique, la pharmacogénétique et la pharmacoéconomie, le système standardisé de recherche des effets secondaires des médicaments appelé Development and rational use of standardised Medical Dictionary for Regulatory Activities (MedDRA) et Standardised MedDRA Queries (SMQs) [1] développé par l'International Conference on Harmonisation (ICH), le développement de la recherche et de la pharmacovigilance dans les pays pauvres en ressources, la pharmacovigilance en matières de vaccins, l'application de la détection précoce des signaux en pharmacovigilance (Early Signal Detection).

C'est du deuxième domaine, celui de l'éthique en recherche médicale que traite cet article. Deux publications actuelles ont résulté des travaux du CIOMS. La première est intitulée Directives Internationales pour la Recherche Biomédicale Incluant des Sujets Humains. Publiées pour la première fois en 1993, ces directives ont été révisées et publiées dans leur nou-

Figure 1

Classification des principaux types de recherches en épidémiologie.

\begin{tabular}{|c|c|c|}
\hline Observationelles & & $\begin{array}{l}\text { Interventionelles } \\
\text { ou expérimentales }\end{array}$ \\
\hline Descriptives & Analytiques & \\
\hline Bases de données & Etudes de cohorte & $\begin{array}{l}\text { e.g. Vaccination, modifications } \\
\text { diététiques, mesures préventives } \\
\text { sur la place de travail }\end{array}$ \\
\hline Registres & Etudes cas-contrôles & \\
\hline Biobanques & Etudes cross-sectionnelles & \\
\hline Dossiers médicaux & Echantillons de groupes (clusters) & \\
\hline
\end{tabular}

velle version en 2002 [2]. Leur but est, à l'usage des investigateurs, des sponsors et des Comités d'éthique évaluant l'adéquation éthique des projets de recherche sur l'être humain, de définir, justifier et édicter les critères éthiques dont ils doivent tenir compte dans l'élaboration et l'exécution des projets de recherche, notamment des essais cliniques de médicaments. S'appuyant sur les principes fondamentaux contenus dans la Déclaration Universelle des Droits de l'Homme (1948) et dans la Déclaration d'Helsinki (1964) de l'Association Mondiale des Médecins et ses révisions successives, ces directives entrent dans le détail de leur application et mise en pratique sur le terrain. Elles sont largement utilisées et respectées dans le monde entier par les Comités d'Ethique qui s'y réfèrent dans leur évaluation des projets qui leur sont soumis de par la loi. Elles ont été traduites en huit langues, et d'autres sont en cours, mais c'est la version en anglais qui demeure l'officielle. Un chapitre de l'Oxford Textbook of Clinical Research Ethics leur est consacré [3].

Plus récemment, ce sont les Directives Internationales Éthiques pour les Études Épidémiologiques datant de 1991 qui ont été révisées ces dernières années et publiées dans leur nouvelle version en 2009 [4]. Un article récent de l'International Journal of Epidemiology de F. Claudot et al. [5] attire l'attention sur la diversité des régulations nationales et des politiques éditoriales des journaux en matière de recherches en épidémiologie, notamment lors d'études observationnelles, et appelle à une harmonisation en la matière. C'est précisément le but poursuivi par ces directives du CIOMS, comme le rappelle M. Cuttini et R. Saraci, directives qui sont actuellement les seules disponibles mondialement [6].

La recherche épidémiologique comprend divers types (Fig. 1). Elle peut être expérimentale ou interventionnelle et se rapproche en ce sens des essais cliniques sur l'être humain et donc partage en partie les mêmes risques tout en portant sur des populations ou des sous-groupes de celles-ci (par exemple les essais de vaccins). Ou bien elle peut être du type observationnel. Dans ce type on distingue deux groupes de formes. Le premier est descriptif utilisant des bases de données, des registres (comme les registres de tumeurs), des biobanques pouvant collecter des prélèvements tissulaires (biopsies, pièces opératoires ou provenant d'autopsie, du sang, du plasma ou du matériel génétique comme 
l'ADN), enfin des dossiers médicaux. Le deuxième groupe d'études observationnelles est analytique et comprend des études de cohortes, des études comparatives dites «cas-contrôles», des études cross-sectionnelles et des études de comparaison de groupes ou clusters (que ce soit des classes d'école, des groupes de travailleurs exposés ou non à des dangers environnementaux sur la place de travail, des échantillons de différentes populations afin de distinguer le rôle de facteurs comportementaux, génétiques, biologiques ou environnementaux, etc.).

\section{Il est impérieux de répéter sans relâche les principes d'éthique médicale - répéter mais aussi surveiller I'application}

Certains pensent que la recherche en épidémiologie de type observationnel est dénuée de dangers. Or, même dans ces études, il faut considérer des effets négatifs [7] non physiques il est vrai, mais du type du tort moral ou d'atteinte aux intérêts privés, tels que: la violation de la confidentialité (vis-à-vis de la famille, de l'entourage, des collaborateurs et/ou de l'employeur sur la place de travail, des assurances-maladie ou des assurances-vie), l'invasion de la sphère privée (par exemple lors d'interviews ou de questionnaires réalisés suite à un incident, médical ou non, dont les sujets de l'étude ne souhaiteraient pas vouloir réveiller le souvenir douloureux ou le révéler à des proches, la stigmatisation et/ou la discrimination d'un sousgroupe d'une population (ethnies, adhérents d'une religion, sujets classés selon leur comportement socioculturel, porteurs d'une affection génétique héréditaire, etc.). Or il faut se rappeler qu'à l'heure du tout électronique, il n'est que trop aisé de fusionner des fichiers électroniques et ainsi d'associer à des données sont associées dans des fichiers séparés ou non. Enfin une nouvelle directive porte sur les conflits d'intérêt.

Confiant que la majorité des chercheurs en épidémiologie se conforment spontanément à ces règles ou ont à cœur d'appliquer ces directives d'éthique médicale, le CIOMS estime toutefois qu'il est important de les rappeler aux anciennes générations et de les enseigner aux nouvelles, et aussi de les mettre en main des membres des commissions d'éthique examinant des protocoles de recherche, membres qui ne sont pas tous versés dans les particularités des recherches en épidémiologie. Ces recherches sont essentielles pour faire avancer nos connaissances en santé publique et leurs résultats devraient même être bien davantage connus du grand public. Ainsi combien de morts auraient pu être épargnés si les gouvernements et les industriels avaient écoutés les cris d'alarme et suivi les conseils des épidémiologistes dans le cas de la catastrophe de l'amiante.

Il ne s'agit pas dans ces directives de mettre des barrières arbitraires aux recherches en épidémiologie. Le CIOMS dans son intitulé comprend les sciences médicales. Or, comme le dit Harry Hopkins: «La recherche dépense pour sauver» [8] que je compléterais en disant: «La science dépense pour sauver des êtres humains et sauver aussi de l'argent pour les générations à venir». Le coût faramineux pour toute l'humanité du scandale de l'amiante est là pour nous le rappeler. Il serait impérieux que des études épidémiologiques prospectives s'attachent vérifier s'il existe ou non un danger pour la santé publique de la dissémination des nouvelles nanoparticules de toutes sortes, comme semblent déjà l'indiquer des articles récents $[9,10]$.

Si les premières règles d'éthiques en matière de recherche médicale sur l'être humain ont été rendues nécessaires suite au Procès de Nuremberg sur les horreurs perpétrées par les Nazis et leurs alliés et ont été édictée dans le Code de Nuremberg (1947), il faut aussi, hélas, rappeler que les plus grands scandales d'atrocités et de conduites non-éthiques ont été sus-

\section{Combien de morts auraient pu être épargnés si les gouvernements et les industriels avaient écoutés les cris d'alarme et suivi les conseils des épidémiologistes dans le cas de la catastrophe de l'amiante}

purement biologiques des données de caractère strictement privé. D'où la nécessité d'établir des règles de codage strictes et de connaître les gardiens des banques de données et de l'existence de clés de codage. Ce thème fait d'ailleurs le sujet de nouvelles directives qui n'existaient pas dans la version précédente. L'une d'elles porte sur l'utilisation de l'Internet dans les études épidémiologiques et une autre sur les échantillons biologiques stockés et des données qui leurs cités par des études, naturellement non soumises à l'époque à des comités d'éthique et contraires à toutes les règles d'éthique médicales, précisément dans le domaine de l'épidémiologie. Que l'on se souvienne du fameux programme de Tuskegee de 1932 à 1972 visant à suivre l'évolution de la syphilis chez des noirs américains non traités et tant d'autres cas passés en revue dans des chapitres du même Oxford Textbook of Clinical Research Ethics cité ci-dessous. Et pire encore 
l'horreur durant la deuxième guerre mondiale des «recherches» menées par Shiro Ishii, responsable japonais de l'Epidemic Prevention Department (Unité 731) de l'Armée japonaise Kwantung, et tous ses nombreux collègues médecins, s'exerçant sur des milliers de Chinois, femmes, enfants et vieillards compris ainsi que des prisonniers de guerre, auxquels les agents infectieux de l'anthrax, de la peste, de la typhoïde, de la paratyphoïde A et B étaient administrés par diverses voies et suivis à divers stades de la maladie par des «autopsies à chaud» [11].

C'est assez dire qu'après les horreurs perpétrées durant et même longtemps après la deuxième guerre mondiale, voire même après la Déclaration Universelle des Droits de l'Homme et la Déclaration d'Helsinki, il est impérieux de répéter sans relâche les principes d'éthique médicale. Répéter mais aussi surveiller l'application de ces directives internationales d'éthique

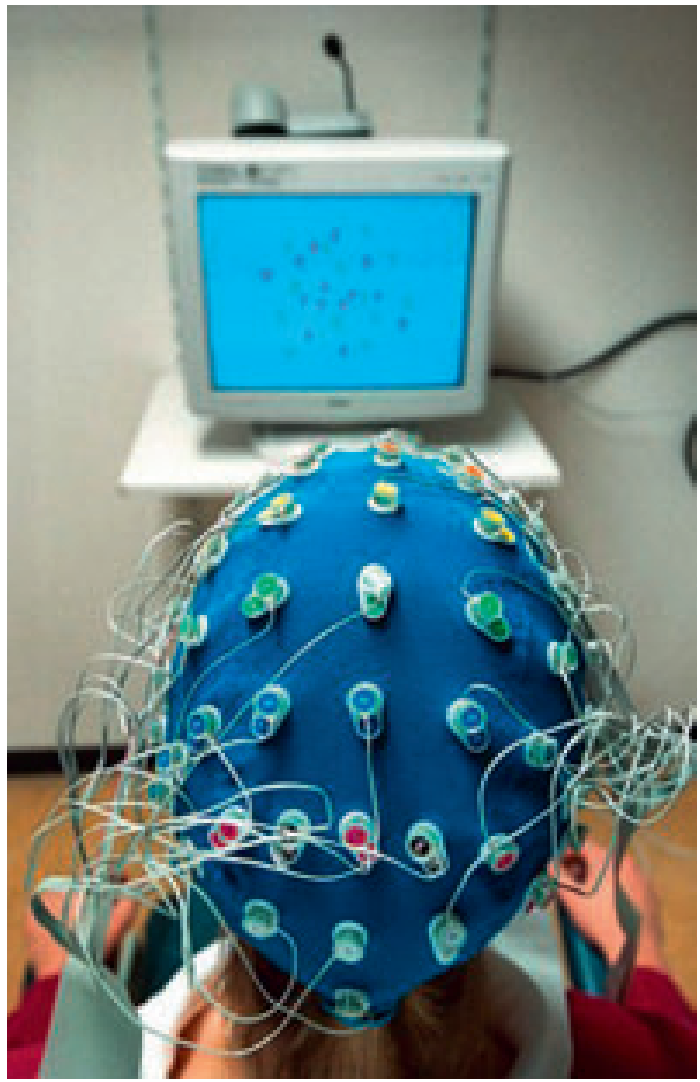

La personne doit toujours rester au centre d'attention de l'éthique en recherche. médicale tels qu'énoncées et décrites dans tous les détails de leur mise en œuvre pratique dans tout projet de recherche, qu'il porte sur des êtres humains isolés ou sur des groupes humains.

\section{Références}

1 SMQs Development and Rational Use of Standardised MedDRA Queries (SMQs) Retrieving Adverse Drug Reactions with MedDRA. CIOMS. 2004; 96 pp.

2 International Ethical Guidelines for Biomedical Research Involving Human Subjects. Prepared by the Council for International Organizations of Medical Sciences (CIOMS) in collaboration with the World Health Organization (WHO). CIOMS. Geneva; 2002; 112 pp.

3 Idänpään-Heikkilä JE, Fluss SS. International Ethical Guidance From the Council for International organizations of Medical Sciences, p.167-73. In: The Oxford Textbook of Clinical Research Ethics. Edited by EJ Emanuel, Ch Grady, RA Crouch, RK Lie, FG Miller and D Wendler. Oxford University Press. 2008.

4 International Ethical Guidelines for Epidemiological Studies. Prepared by the Council for International Organizations of Medical Sciences (CIOMS) in collaboration with the World Health Organization (WHO). CIOMS. Geneva. 2009; 128 pp.

5 Claudot F, Alla F, Calvez T, Coudane H, Bonati-Pellié C. Ethics and observational studies in medical research: various rules in a common framework. Int J Epidemiol. 2009;38(4):1104-8.

6 M Cuttini, Saraci R. Commentary: Can we facilitate the ethical approval of international observational studies? Int J Epidemiol. 2009;38(4):1108-9.

7 Capron A. Protection of research subjects: do special rules apply in epidemiology? J Clin Epidemiol 1991;44(Suppl.I): 818-98.

8 Citation par William C. Gibson. The cost of not doing health research, In: Humanitarian Medicine. 2005; p.233.

9 Poland CA, Duffin R, Kinloch I, Maynard A et al. Carbon nanotubes introduced into the cavity of mice show asbestos-like pathogenicity in a pilot study. Nat Nanotechnol. 2008;2(7):423-8.

10 Ryman-Rasmussen JP, Cesta MF, Brody AR et al. Inhaled carbon nanotubes reach the subpleural tissue in mice. Nat Nanotechnol. 2009;4(11):747-51. Comment on p.708-10.

11 Tsuchiya T. The Imperial Japanese Atrocities and Its Enduring Legacy in Japanese Research Ethics p.31-45. In: The Oxford Textbook of Clinical Research Ethics. Oxford University Press. 2008:827 pp. 